\title{
Nicht nur die Mitarbeiter müssen lernen, auch die Organisation
}

\author{
Jörg Longmuß und Manfred Mühlfelder
}

Agiles Lernen im Unternehmen braucht, wenn es mehr als ein einzelnes Ereignis sein soll, seinen eigenen Platz in den internen Abläufen des Unternehmens. Hier ein Überblick über Erfahrungen, die in den Betriebsprojekten gemacht wurden, und die Schlussfolgerungen, die daraus gezogen werden können. Sie zeigen, in welche Richtung ein Unternehmen Entwicklungen erlauben und auch vorantreiben muss, um agiles Lernen längerfristig und in größerem Umfang zu ermöglichen.

Zum einen braucht es einen Raum, physisch wie zeitlich und sinnbildlich, in dem die Lernenden konzentriert arbeiten können. Zum anderen wird die Integration des Lernens in den Arbeitsprozess in vielen Fällen ein neues Zusammenspiel von Personalentwicklung und Fachabteilungen erfordern. Das betrifft sowohl die Verteilung von Ressourcen als auch die Abstimmung untereinander. Beides wird im Folgenden näher beschrieben:

- die Vor- und Nachbereitung des Lernprojekts und der Aufgaben in Kap. 11,

- Anforderungen an eine Qualitätssicherung in Kap. 12,

- die Qualifizierung der Begleiter in Kap. 13 und

- eine zweckmäßige Infrastruktur in Kap. 14.

Weitere Punkte, die für die Einführung von agilem Lernen in einem Unternehmen wichtig sind, werden in anderen Kapiteln näher beschrieben.

J. Longmuß $(\bowtie)$

SUSTAINUM - Institut für zukunftsfähiges Wirtschaften Berlin, Berlin, Deutschland

E-Mail: j.longmuss@sustainum.de

M. Mühlfelder

SRH Fernhochschule - The Mobile University, Riedlingen, Deutschland

E-Mail: manfred.muehlfelder@mobile-university.de 


\subsection{Lernen „stört ${ }^{\prime \prime}$}

Es gibt ein strukturelles Spannungsverhältnis zwischen einem hochverdichteten Arbeitsprozess und gezieltem Lernen am Arbeitsplatz. Lernen ist in einem solchen Kontext immer auch störend, weil die Arbeitskraft nicht lückenlos aktuellen Arbeitsaufgaben zugeteilt werden kann. Wer lernt, braucht Ruhe und die Möglichkeit, sich zu fokussieren. In allen Betriebsprojekten war es wichtig, dies sicherzustellen.

In vielen Fällen wird hier ein „Pauschaler Lernfreiraum“ zielführend sein, d. h. eine fest vereinbarte Zeit und andere Ressourcen über das hinaus, was für das Erarbeiten einer unmittelbar praxistauglichen Lösung zwingend notwendig ist. Es war z. B. unter anderem bei MAN nicht möglich, das Bearbeiten von Lernaufgaben über eine unmittelbare Problembearbeitung hinaus durchzusetzen, weil die Mitglieder des Teams ohnehin schon regelmäßig Überstunden gemacht hatten.

Ein pauschaler Lernfreiraum hingegen begünstigt entdeckendes Lernen und eine nachhaltige Beschäftigung mit dem Lerngegenstand. So kostet er nicht einfach Zeit, sondern bringt inhaltliche Tiefe und einen fachlichen Mehrwert. In mehreren Unternehmen wurden so Möglichkeiten einer Prozessverbesserung entdeckt. Der Umfang eines solchen Lernfreiraums muss für jedes Lernprojekt (wie für jede Art von Projekten) neu bestimmt werden.

Als zentrale Anforderungen an einen solchen Lernfreiraum erwiesen sich ein geschützter äußerer Rahmen, eine allseitige Fehlertoleranz und eine positive Feedbackkultur.

\section{Ein äußerer Rahmen}

Zunächst ist es wichtig, eine Struktur zu schaffen, die den Lernenden Sicherheit gibt. Dazu gehören:

- Ein gesicherter Zeitraum. Die Lernenden müssen ausreichend Zeit zur Verfügung haben und sich darauf auch verlassen können. Ein langfristig wiederkehrender Termin, z. B. immer donnerstags von 10:00 bis 16:00 Uhr, hat sich in vielen Fällen bewährt. Diese Zeit steht beim Lernen direkt im Unternehmen trotzdem oft unter Druck, weil es relativ einfach ist, die Lernenden kurzfristig einer anderen Aufgabe zuzuteilen (anders als beispielsweise bei einem Seminar außerhalb). Dies war in mehreren Lernszenarien und Unternehmen z. B. bei MAN und Bayer teilweise problematisch. Es wird zwar immer wieder dringende Anforderungen aus dem betrieblichen Alltag geben, bei denen das Lernen zurückstehen muss. Die Schwelle hierfür sollte allerdings sehr hoch sein: Mitarbeiter, die an einem externen Seminar teilnehmen, werden auch nur in Notfällen zurückgerufen. Damit dies auch für Lernphasen im Unternehmen gilt, müssen die Vorgesetzten den Zeiträumen nicht nur zustimmen, sondern das nötige Maß an Lernzeit auch schützen gegen Anforderungen aus dem eigenen und aus anderen Bereichen.

- Eine unterstützende Infrastruktur. Dazu sind als erstes Lern- und Arbeitsräume nötig, in denen ein Team zusammenkommen und ungestört arbeiten kann. In den meisten 
Betriebsprojekten wurde nicht in Vollzeit, sondern in mehr oder weniger umfangreicher Teilzeit gelernt. Dabei hat es sich positiv ausgewirkt, wenn Arbeitsmittel, z. B. Moderationswände oder beschriftete Flipcharts, im Raum bleiben und beim nächsten Treffen hervorgeholt werden konnten. Zu dieser Infrastruktur gehören auch die Tools zur Kommunikation im Team (s. Kap. 14) und ggf. weitere Ressourcen, z. B. ProjektmanagementSoftware.

- Zugänge zu Wissen. Das bezieht sich zum einen auf Personen im Unternehmen, die fachlich einschlägige Kenntnisse und Erfahrungen haben und dem Team weiterhelfen können, ohne deshalb fachliche Begleiter zu sein. Zum anderen sind damit weitere Unterlagen z. B. zum Arbeitsprozess gemeint sowie Auskünfte aus fachfremden Bereichen, z. B. Controlling, Behördenkommunikation, Arbeitsschutz, ggf. auch aus der Organisationsentwicklung oder dem Management.

\section{Allseitige Fehlertoleranz für ein schnelles und gleichzeitig nachhaltiges Lernen}

Schnelles, selbstorganisiertes Lernen schließt notwendigerweise ein, dass die ersten Ergebnisse noch nicht perfekt sind und auch immer wieder fachliche Fehler gemacht werden. Umgekehrt würde ein zu großer Druck, auf Anhieb ein makelloses Ergebnis produzieren zu müssen, zu Verunsicherung führen, ein aktives Experimentieren bremsen und wenig nachhaltige Kompetenzentwicklung erreichen. Dies erfordert jedoch von allen Beteiligten, d. h. von den Auftraggebern und dem Team, den einzelnen Lernern wie dem Management, Akzeptanz für eine Annäherung an die Lösung in kleinen Schritten und das frühzeitige Experimentieren mit Teillösungen. Gefordert ist besonders:

- Vom Team: Den Anspruch an die eigene Arbeit nicht absolut zu setzen, d. h. auszuhalten, dass es nicht unbedingt in jeder Etappe eine einwandfrei umsetzbare Lösung gibt, sondern vielleicht auch nur erste, tastende Schritte. Es ist nicht als Scheitern, sondern als als Lernschritt zu sehen, wenn nachgebessert werden muss. Das agile Lernen verläuft iterativ, das heißt, indem Prozesse durch Wiederholungen optimiert werden.

- Von der Planung: Erfolg (wie Misserfolg) findet in kleinen Schritten statt. In einem der untersuchten Unternehmen war der erste Iterationsschritt so groß, dass die Motivation des Teams zerbrach, als sich zeigte, dass dieser Schritt in die falsche Richtung gegangen worden war. Um dies zu vermeiden, müssen die Lernschritte klein sein und als Experimente begriffen werden.

- Vom Auftraggeber: Die Beurteilung eines Ergebnisses nicht nach Reifegrad im Sinne einer vollständigen Lösung und fachlichen Fehlerlosigkeit, sondern daraufhin, ob das Ergebnis einen erkennbaren Erkenntnisfortschritt bringt in Richtung auf das Lernziel. Im Sinne einer Experimentier-Kultur kann ein solcher Fortschritt auch durch ein fachlich unzureichendes Zwischenergebnis erreicht werden, wenn dieses neue Erkenntnisse bringt und zu einem Lernfortschritt geführt hat. In einem Projekt wurde ein Zwischenergebnis vom Bereichsleiter zuerst grundsätzlich abgelehnt - um dem Team dann zu erklären, aus welchen Gründen im Unternehmen solche Zurückweisungen vorkommen 
können, wie man sich darauf vorbereiten kann und welche Strategien es gibt, sie zu bewältigen. Im Ergebnis hatte das Team sehr viel über das Agieren auf der ManagementEbene eines großen Unternehmens gelernt.

Das bedeutet ausdrücklich nicht, dass Auftraggeber nicht auf ein fachlich gutes Ergebnis achten sollen. Es bedeutet vielmehr, ein Verständnis dafür zu entwickeln, dass Lernen ein langwieriger Prozess sein kann. Dazu gehört auch, die Verzögerung einer vielleicht schnelleren zugunsten einer besseren/nachhaltigeren Lösung zu akzeptieren. Und es bedeutet, dass den Lernaufgaben genug zeitlichen Spielraum für diesen Prozess eingeräumt werden muss.

\section{Eine positive Feedback-Kultur im Unternehmen}

Eine positive Feedback-Kultur lässt sich beschreiben als eine Haltung im Unternehmen, Feedback konstruktiv, bereitwillig und offen zu geben sowie die Bereitschaft, damit produktiv umzugehen - und zwar in alle Richtungen, d. h. horizontal und vertikal sowie von den Organisationsrändern zum Zentrum und vom Zentrum nach außen. Dies ist beim agilen Lernen vor allem im Review und in der Retrospektive bedeutsam. Das Feedback im Review ist nicht nur ein Signal zum Weiterarbeiten, sondern darüber hinaus

- eine Aussage über die Relevanz der Lernergebnisse für die Organisation,

- eine fachliche Anerkennung: Die inhaltliche Güte einer Lösung wird bestätigt,

- im Projektabschluss eine Form von Anerkennung „Du kannst, was hier verlangt wird“.

Dieses Feedback im weiteren Sinne beinhaltet, dass die Vorgesetzten und ggf. die fachlichen Begleiter nicht nur den Rahmen bereitstellen, sondern sich auch selbst stärker auf Lernprozess und -inhalt einlassen bzw. dies an geeignete Personen delegieren. Das erfordert mehr Aufmerksamkeit und auch Zeit, als bei externen Schulungen seitens der Vorgesetzten meist aufgewandt wird. Grundsätzlich sollte eine solche Aufmerksamkeit von Führungskräften auch zu erwarten sein. Gleichzeitig wird es in vielen Unternehmen und Fachbereichen für eine Übergangszeit Unterstützung für die Führungskräfte brauchen, sich in eine solche Rolle hineinzufinden.

\subsection{Weiterbildung zwischen Personalentwicklung und Fachabteilung}

Agile Lernprojekte sind immer kontextgebunden. Das erfordert bei einer breiteren Anwendung von agilem Lernen eine enge inhaltliche Abstimmung zwischen Fachabteilungen und der Personalentwicklung. Die Fachabteilungen müssen ihren Bedarf an Kompetenzentwicklung präzise deutlich machen und agiles Lernen fachlich begleiten; die Personalentwicklung muss zielgenaue Angebote bereitstellen. Eine so enge Zusammen- 
arbeit ist allerdings nicht überall der Fall. Für viele Unternehmen besteht hier noch ein Bedarf an Organisationsentwicklung.

\section{Wo kommen die Begleiter her?}

Je umfangreicher agiles Lernen eingesetzt wird, desto mehr Zeit müssen die fachlichen und methodischen Begleiter für ihre Rollen in den Lernprojekten aufwenden. Gleichzeitig wird aber kein Vorgesetzter langfristig Mitarbeiter abstellen können, um den Weiterbildungsbedarf anderer Abteilungen abzusichern.

Mittelfristig entstehen hier neue Aufgaben in der Personalentwicklung sowie ein verändertes Aufgabenprofil. Geklärt und gelöst werden muss,

- wie die zu erwartenden Aufwände transparent gemacht werden können,

- wer auf Dauer die Ressourcen (Begleiter, Räume, Software etc.) organisiert,

- auf welche Kostenstellen die methodischen und fachlichen Begleiter abgerechnet werden,

- wer mittelfristig die Begleitung trägt, d. h. ob und wie weit externe Begleiter beauftragt oder interne Begleiter für diese Aufgabe freigestellt und geschult werden.

So entstand in einem der am Forschungsprojekt beteiligten Unternehmen, in dem es einen Bedarf an umfangreichen agilen Kompetenzentwicklungen gab, ein Dilemma. Auf der einen Seite konnte der Vorgesetzte einer Fachabteilung die Aufwände für die Begleitung nicht mehr mit der aktuellen Kapazitätsplanung vereinbaren. Auf der anderen Seite legten die Mitarbeiter dieser Abteilung Wert darauf, dass das Lernprojekt weiterhin von internen Fachleuten gestützt werden sollte, weil diese hochwertigen Fachinput liefern konnten.

Ausweg aus einem solchen Dilemma kann die Aufnahme der Begleitung in die Zielvereinbarungen und die Kapazitätsplanung der Fachleute sein, Eine andere Option ist die langfristige Einbeziehung externer Fachbegleiter, wie es bei Inhouse-Seminaren auch geschieht.

\section{Ein Platz in der betrieblichen Weiterbildung}

In den Unternehmen gibt es für die Anerkennung von betrieblichen Weiterbildungen meist ein etabliertes System. Es bestimmt, in welchem Umfang die Teilnahme an Seminaren erwartet und gefördert wird sowie mit welcher Wertigkeit Weiterbildungen anerkannt werden. Entsprechende Zertifizierungen sind ein wichtiger Bestandteil für Personalentwicklung, Personalplanung und Karriereplanung. Bei agilen Lernprojekten stellen sich hier Fragen, die in jedem Fall zu klären sind:

- Welcher Lernumfang eines agilen Lernprojekts entspricht welchem Seminarumfang? Da auch praktische Aufgaben bearbeitet werden, liefern die reinen Stundenzahlen nicht unbedingt einen sinnvollen Maßstab.

- Wie kann das im agilen Lernen angelegte Erfahrungslernen auf hoher Kompetenzstufe angemessen bewertet werden? 
- Wer entscheidet über die Einstufung und wie soll zertifiziert/anerkannt werden? In mehreren Fällen wurden die externen Fachbegleiter um ein Zertifikat gebeten, weil dies in den internen Regularien nicht vorgesehen war.

- Wie kann sich agiles Lernen in die interne Weiterbildung einfügen?

In größeren Unternehmen kommt es vor, dass die Weiterbildung an ein Tochterunternehmen in Form einer Akademie ausgelagert wird, welche für ihre Erlöse selbst verantwortlich ist - so auch in einem der Unternehmen, in denen das agile Lernen umgesetzt wurde. Diese Akademie wollte von sich aus nicht die Entwicklungskosten für den umfassenden Aufbau eines Angebots „Agiles Lernen“ tragen.

Gleichzeitig war die internationale Managementebene, die einen solchen Auftrag hätte erteilen können, zu weit von den Erfahrungen der Fachabteilung entfernt, in der die positiven Erfahrungen gemacht wurden. Von sich aus war die Fachabteilung aber nicht in der Lage, einen solchen Aufbau in Auftrag zu geben. Im Ergebnis wurde das agile Lernen nicht so im Unternehmen ausgerollt, wie es die guten Erfahrungen im Pilotprojekt nach Ansicht aller Beteiligten nahegelegt hätten. Hier wäre die Einbeziehung der Personalentwicklung von Anfang an sicher eine gute Vorgehensweise gewesen.

\subsection{Was braucht es also?}

Zusammenfassend sind die wichtigsten Gestaltungsbedarfe in der Unternehmensorganisation für agiles Lernen:

- Unterstützung: Zusätzlich zu den Begleitern des Teams sollte auch den Vorgesetzten/ Auftraggebern Hilfestellung angeboten werden zur Schaffung von Rahmenbedingungen für agile Lernprojekte.

- Ressourcen: Für die fachlichen und methodischen Begleiter sollten Ressourcen freigegeben werden, die Berücksichtigung dieser Aufgaben muss in den Zielvereinbarungen formuliert im Jahresbudget geplant sein.

- Anerkennung: Die Teilnahme an einem agilen Lernprojekt sollte in das interne Anerkennungssystem für Weiterbildungen eingespeist werden, dazu sollten Äquivalenzen gegenüber herkömmlichen Seminaren evaluiert werden.

- Vernetzung: Die Personalentwicklung sollte bereits früh in Konzeptionierung und Gestaltung des agilen Lernens im Unternehmen einbezogen werden, um eine Einbindung agiler Lernangebote in das gesamte Weiterbildungsangebot anzustoßen. 
Open Access Dieses Kapitel wird unter der Creative Commons Namensnennung - Weitergabe unter gleichen Bedingungen 4.0 International Lizenz (http://creativecommons.org/licenses/ by-sa/4.0/deed.de) veröffentlicht, welche die Nutzung, Vervielfältigung, Bearbeitung, Verbreitung und Wiedergabe in jeglichem Medium und Format erlaubt, sofern Sie den/die ursprünglichen Autor(en) und die Quelle ordnungsgemäß nennen, einen Link zur Creative Commons Lizenz beifügen und angeben, ob Änderungen vorgenommen wurden. Wenn Sie das Buch oder Teile daraus remixen, verändern oder anderweitig direkt darauf aufbauen, dürfen Sie Ihre Beiträge nur unter derselben Lizenz wie das Original verbreiten.

Die in diesem Kapitel enthaltenen Bilder und sonstiges Drittmaterial unterliegen ebenfalls der genannten Creative Commons Lizenz, sofern sich aus der Abbildungslegende nichts anderes ergibt. Sofern das betreffende Material nicht unter der genannten Creative Commons Lizenz steht und die betreffende Handlung nicht nach gesetzlichen Vorschriften erlaubt ist, ist für die oben aufgeführten Weiterverwendungen des Materials die Einwilligung des jeweiligen Rechteinhabers einzuholen. 